\title{
氭基取代的螺芴氧杂葱衍生物：激基复合物发光与性质调控
}

\author{
曹洪涛 ${ }^{\dagger,}, \quad$ 李波 ${ }^{\dagger}, a$ 万俊 ${ }^{a}$ 余涛 $^{a}$ 解令海 ${ }^{*, a} \quad$ 孙辰 $^{b}$ \\ 刘玉玉*,a王锦 ${ }^{*}$ 黄维 $*, a, c$ \\ ( ${ }^{a}$ 南京邮电大学信息材料与纳米技术研究院 有机电子与信息显示国家重点实验室培育基地 南京 210023) \\ ( ${ }^{b}$ 西班牙马德里高等研究中心纳米研究所 马德里 28049) \\ ( ${ }^{c}$ 西北工业大学柔性电子前沿科学中心 西安 710072)
}

\begin{abstract}
摘要 热活化延迟荧光分子因具有高效发光、价格低廉等优点, 在发展有机发光二极管方面显示出巨大潜力. 与单分 子相比，激基复合物容易实现小的单线态-三线态能隙差，在开发延迟苂光材料方面备受关注. 然而，相应受体材料的 种类仍较为稀少，且激基复合物延迟苂光性质与受体材料结构之间的关系还需深入探讨. 本工作设计合成出两个新型 的基于螺荡氧杂葱的电子受体材料(CNSFDBX 和 DCNSFDBX). 结果表明, 它们与给体材料 TCTA 掺杂后均呈现激基 复合物发射, 其中 TCTA:DCNSFDBX 掺杂体系显示更高的发光效率, 其原因归结为双氰基取代使得 DCNSFDBX 具有 更强的接收电子的能力. 该工作为开发新型电子受体材料用于激基复合物延迟苂光提供了思路.
\end{abstract}

关键词＼cjkstart螺芴氧杂葱; 激基复合物发光；热活化延迟苂光; 空间位阻; 氧基取代

\section{Cyano-substituted Spiro[fluorine-9,9'-xanthene] Derivatives: Exciplex Emission and Property Manipulation}

\author{
Cao, Hongtao ${ }^{\dagger, a} \quad \mathrm{Li}, \mathrm{Bo}^{\dagger, a} \quad{\text { Wan, } \mathrm{Jun}^{a} \quad \mathrm{Yu}^{\circ}, \mathrm{Tao}^{a} \quad \text { Xie, Linghai }}^{*, a} \quad$ Sun, $\mathrm{Chen}^{b}$ \\ Liu, Yuyu ${ }^{*, a}$ Wang, Jin $^{a}$ Huang, Wei*,a,c \\ ( ${ }^{a}$ Key Laboratory for Organic Electronics and Information Displays \& Institute of Advanced Materials (IAM), \\ Jiangsu National Synergetic Innovation Center for Advanced Materials (SICAM), \\ Nanjing University of Posts \& Telecommunications (NUPT), Nanjing 210023, China) \\ $\left({ }^{b}\right.$ Madrid Institute for Advanced Studies in Nanoscience, IMDEA Nanociencia Calle Faraday 9, \\ Ciudad Universitaria de Cantoblanco 28049, Spain) \\ ( ${ }^{c}$ Frontiers Science Center for Flexible Electronics (FSCFE), Shaanxi Institute of Flexible Electronics (SIFE) \& \\ Shaanxi Institute of Biomedical Materials and Engineering (SIBME), \\ Northwestern Polytechnical University (NPU), Xi'an 710072, China)
}

Abstract Thermally activated delayed fluorescence (TADF) molecules have great potential in developing organic light-emitting diodes (OLEDs) because of their efficient emission and low price. Compared to pure-molecules, exciplex systems are drawing much attention since they can realize small singlet-triplet energy splitting $\left(\Delta E_{\mathrm{ST}}\right)$ more easily for TADF. However, the species and molecular design systems of electron-acceptors for exciplex-TADF are still limited even though some acceptors have been reported. In addition, the relationship between TADF properties and the structures of acceptors requires further investigations. Herein, we report the design and synthesis of two novel spiro[fluorine- $9,9^{\prime}$-xanthene]-based acceptors (CNSFDBX and DCNSFDBX) for achieving exciplex-emissions by using tris(4-carbazoyl-9-ylphenyl)amine (TCTA) as a donor. The photoluminescence measurements suggest that both of the doping-systems (TCTA:CNSFDBX and TCTA:DCNSFDBX) possess exciplex emissions. Whereas, it is observed that the TCTA:DCNSFDBX system displays higher photoluminescence quantum yield and electroluminescence efficiency than TCTA:CNSFDBX. For better explaining this phenomenon, we perform low-temperature fluorescence and phosphorescence spectra investigations. The experimental results show that the TCTA:DCNSFDBX system exhibits smaller $\Delta E_{\mathrm{ST}}$ values $(0.12 \mathrm{eV})$ than TCTA:CNSFDBX $(0.46 \mathrm{eV})$. This results indicate that the reverse intersystem crossing from non-radiative triplet states $\left(\mathrm{T}_{1}\right)$ to radiative singlet states $\left(\mathrm{S}_{1}\right)$ and TADF processes can be realized more easily in the TCTA:DCNSFDBX system. Moreover, the electrochemical measurements and theoretical calculations suggest that the lowest unoccupied molecular orbital (LUMO) level of DCNSFDBX

*E-mail: iamlhxie@njupt.edu.cn; iamyyliu@njupt.edu.cn; iamwhuang@njupt.edu.cn

$\uparrow$ These authors contributed equally to this work

Received March 31, 2020; published June 3, 2020.

Supporting information for this article is available free of charge via the Internet at http://sioc-journal.cn.

Project supported by the National Natural Science Foundation of China (Nos. 61605090, 61604081, 61604076, 21774061, 91833306), the Natural Science Foundation of Jiangsu Province (BK20190090, BK20180751), the Six Peak Talents Foundation of Jiangsu Province (XCL-CXTD-009) and the Priority Academic Program Development of Jiangsu Higher Education Institutions (PAPD, YX030003).

项目受国家自然科学基金(Nos. 61605090, 61604081, 61604076, 21774061, 91833306)、江苏省自然科学基金(BK20190090, BK20180751)、江苏省 “六 大人才高峰”创新人才团队项目(XCL-CXTD-009)和江苏省高校优势学科建设工程(YX030003)资助. 
$(-2.86 \mathrm{eV})$ is lower than that of CNSFDBX $(-2.47 \mathrm{eV})$. This situation implies that DCNSFDBX possesses stronger electron-accepting ability than CNSFDBX with the help of dicyano-substitution. Furthermore, the TCTA:DCNSFDBX system displays larger driving force $(0.39 \mathrm{eV})$ than TCTA:CNSFDBX $(0.22 \mathrm{eV})$ in their exciplex-formation processes, suggesting the exciplex-emission (TCTA:DCNSFDBX) can be achieved more easily. Therefore, the higher exciplex-emission efficiencies of the TCTA:DCNSFDBX system are attributed to the stronger electron-acceptability of DCNSFDBX through dicyanosubstitution and larger driving force in its exciplex emission process. This work provides a route to further development of new electron-acceptors for exciplex-TADF.

Keywords spiro[fluorine-9,9'-xanthene]; exciplex emission; thermally activated delayed fluorescence; steric hindrance; cyano-substitution

\section{1 引言}

有机发光二极管 (organic light-emitting diodes, OLEDs)因具有高分辨率、全色发光、柔性可弯曲等优 势而在平板显示和照明领域显示出巨大应用潜力 ${ }^{[1-9]}$. 在发展高性能 OLEDs 的进程中, 重金属磷光配合物(如 铱、铂等配合物)已被广泛设计、合成和用于器件的制 备 ${ }^{[10-12]}$. 这是因为这类材料凭借重金属原子的旋轨耦合 作用而显示出有效的磷光发射, 对实现 OLEDs 的高效 发光具有至关重要的作用 ${ }^{[13-15]}$. 然而铱、铂等重金属较 为稀缺, 直接导致这些磷光配合物具有较高的制备成 本, 不利于 OLEDs 实现价格低廉的商业化应用. 因此, 寻找廉价的高效发光材料成为一个亟待解决的问题.

热活化延迟苂光(thermally activated delayed fluorescence, TADF) 是激子通过最低三重激发态 (triplet state, $\mathrm{T}_{1}$ )反系间审越到最低单重激发态(singlet state, $\mathrm{S}_{1}$ ) 再进行辐射跃迁的一种发光现象 ${ }^{[16-18]}$. TADF 材料可避 免铱、铂等重金属原子的引入, 通过小分子的构建便可 实现价格低廉和高效发光的目标 ${ }^{[19-21]}$. 因此, TADF 分 子已成为制备高效发光器件的一种重要选择. 在开发 $\mathrm{TADF}$ 分子方面, 激基复合物(exciplex)型 TADF 材料因 前线分子轨道分布在不同分子结构上而更容易实现较 小的单线态-三线态能隙差 $\left(\Delta E_{\mathrm{ST}}\right)$ 和 TADF 发射, 因此备 受科研工作者的关注 ${ }^{[22,23]} .2012$ 年 Adachi 课题组 ${ }^{[24]}$ 将 4,4',4"'-tris[3-methylphenyl(phenyl)amino]triphenylamine ( $m$-MTDATA) 和 tris-[3-(3-pyridyl)mesityl]borane (3TPYMB) 分别作为电子给体和受体材料, 实现了 exciplex-TADF 发射, 对应的电致发光外量子效率 (external quantum efficiency, $\eta_{\mathrm{ext}}$ ) 为 $5.4 \%$. 后来, 人们开 发出更多的电子受体材料或主体材料来改善 exciplex-TADF 的电致发光效率. 如 $\mathrm{Li}$ 等 ${ }^{[25]}$ 采用 2,5,8-tris(4-fluoro-3-methylphenyl)-1,3,4,6,7,9,9b-heptaaz aphenalene (HAP-3MF) 作为受体材料, 将 exciplex-TADF 的外量子效率提升到 $11.3 \%$; 后来, Zhang 等 ${ }^{[26]}$ 采用 triazine/carbazole hybrid molecule (DPTPCz) 作为受体材料, 获得了 $15.4 \%$ 的外量子效率, $45.7 \mathrm{~cd} \cdot \mathrm{A}^{-1}$ 的电流效率 (current efficiency, $\eta_{\mathrm{c}}$ ) 和 $47.9 \mathrm{~lm} \cdot$ $\mathrm{W}^{-1}$ 的功率效率 (power efficiency, $\eta_{\mathrm{p}}$ ). 截止目前, exciplex-TADF 的发光性能已经得到了很大的改善, 部分器 件的 $\eta_{\mathrm{ext}}$ 值已经超过 $20 \%{ }^{[27,28]}$, 然而相应受体材料的种 类仍较为稀少, 且激基复合物延迟苂光性质与受体材料
结构之间的关系还需深入探讨.

最近, 我们设计开发出基于螺荡氧杂葱 (spiro[fluorine-9,9'-xanthene], SFX) 的新型电子受体材 料, 实现了 exciplex-TADF 发射 ${ }^{[29,30]}$. 但是, 相应的发光 性能有待提升, 而且 exciplex-TADF 性能与 SFX 螺环结 构之间的关系仍需深入挖掘. 因此, 进一步设计开发出 新型 SFX 基电子受体材料, 对于丰富 exciplex-TADF 的 电子受体材料体系及探索受体材料的性能与其结构之 间的关系具有重要作用. 本文对 SFX 结构进行一定修 饰, 设计并合成出两个新型电子受体材料 2-carbonitrilespiro[fluorene-9,9'-dibenzoxanthene] (CNSFDBX), 2,7-dicarbonitrile-spiro[fluorene-9,9'-dibenzoxanthene] (DCNSFDBX); 将 tris(4-carbazoyl-9-ylphenyl)amine (TCTA)作为给体材料, 实现了 exciplex-TADF; 通过光 物理性能测试和器件制备, 对所得到的 exciplex-TADF 性能与受体材料结构之间的关系进行了研究.

\section{2 结果与讨论}

\section{1 分子 CNSFDBX 和 DCNSFDBX 的合成}

我们基于课题组前期的一锅法工作基础 ${ }^{[31-33]}$, 从已 制备的小分子 2-溴螺荡苯并氧杂葱(2-bromo-spiro- [fluorene-9,9'-dibenzoxanthene], BrSFDBX)和 2,7-二溴螺芴 苯并氧杂葱 (2,7-dibromo-spiro[fluorene-9,9'-dibenzoxanthene], DBrSFDBX)出发, 采用一步反应(图式 1)快捷 地合成了目标分子 CNSFDBX 和 DCNSFDBX, 产率分 别为 $85 \%$ 和 $78 \%$.

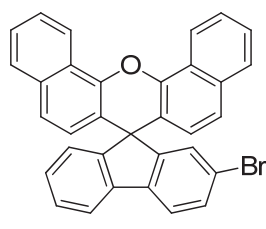

BrSFDBX

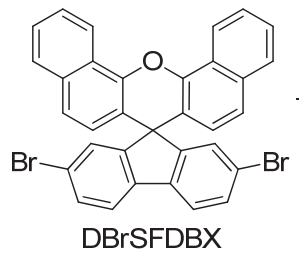

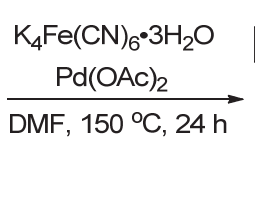

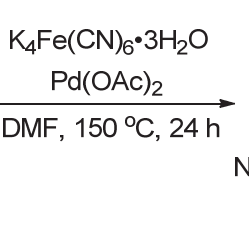

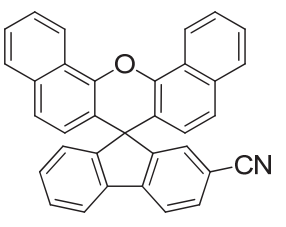

CNSFDBX
图式 1 化合物 CNSFDBX 和 DCNSFDBX 的合成路线

Scheme 1 Synthetic routes of compounds CNSFDBX and DCNSFDBX 
随后我们利用柱色谱操作对目标产物进行了分离 与提纯, 并经过 ${ }^{1} \mathrm{H} N M R,{ }^{13} \mathrm{C} \mathrm{NMR}, \mathrm{GC}-\mathrm{MS}$ 及元素分析 等测试进行了结构确认(谱图信息见 Supporting Information 部分, 图 $\mathrm{S} 1 \sim \mathrm{S} 4)$, 其具体合成信息见实验部分.

\section{2 吸收与发射光谱表征}

我们对单体分子和掺杂体系的薄膜分别进行了吸 收和发射光谱的测试, 结果列于图 1 中.

图 $1 \mathrm{a}$ 和 $1 \mathrm{~b}$ 显示, 掺杂体系 TCTA:CNSFDBX 和 TCTA:DCNSFDBX 与各自对应的单体分子相比, 表现 出相似的吸收特征。这表明, TCTA:CNSFDBX 和 TCTA:DCNSFDBX 在外界光源的激发作用下并未形成 新的基态. 图 $1 \mathrm{c}$ 和 $1 \mathrm{~d}$ 显示, 掺杂体系 TCTA:CNSFDBX 和 TCTA:DCNSFDBX 在外界光源的激发作用下展现出 与各自单体不同的发射光谱. 其中, TCTA:CNSFDBX 的 发射峰位于 $441 \mathrm{~nm}$, 比对应的单体 CNSFDBX 的发射峰 (398 nm)红移 $43 \mathrm{~nm}$; 而 TCTA:DCNSFDBX 的发射峰位 于 $529 \mathrm{~nm}$, 比对应单体 DCNSFDBX 的发射峰 $(434 \mathrm{~nm})$ 红移 $95 \mathrm{~nm}$. 这表明掺杂体系 TCTA:CNSFDBX 和 TCTA:DCNSFDBX 在外界光源的激发作用下形成了与 单体不同的激发态。根据文献报道 ${ }^{[34,35]}$ 可知, TCTA:CNSFDBX 和 TCTA:DCNSFDBX 薄膜的发射来


源于激基复合物发射.

\section{3 室温与变温荧光衰减曲线分析}

随后，我们对单体和掺杂体系的薄膜进行了苂光 衰减曲线测试, 相关结果列于图 2 和表格 $\mathrm{S} 1 \sim \mathrm{S} 2$ 中.

图 $2 \mathrm{a}$ 和表格 $\mathrm{S} 1$ 显示, 单体分子 TCTA, CNSFDBX 和 DCNSFDBX 薄膜具有双组分衰减特征，其寿命分 别为 $0.9 \mathrm{~ns}(90 \%)$ 和 $7.2 \mathrm{~ns}(10 \%), 1.7 \mathrm{~ns}(49 \%)$ 和 $9.2 \mathrm{~ns}$ $(51 \%), 1.1 \mathrm{~ns}(27 \%)$ 和 $7.8 \mathrm{~ns}(73 \%)$. 根据文献报道 ${ }^{[26,36]}$ 可知, TCTA, CNSFDBX 和 DCNSFDBX 的短寿命(衰 减)组分 (分别为 $0.9 、 1.7 、 1.1 \mathrm{~ns}$ ) 来源于瞬时苂光, 而 长寿命(衰减)组分(分别为 $7.2 、 9.2 、 7.8 \mathrm{~ns}$ ) 则可能是因 单体分子在固态堆积状态下的相互作用造成的. 这种 相互作用可能会引起分子发射光谱出现 “宽化”、“尖 峰” 等现象，或者形成 “excimer” 发射，进而导致其 苂光衰减曲线出现双组分寿命特征. 图 $2 b$ 和表格 S1 显示, 掺杂体系 TCTA:DCNSFDBX 比 TCTA:CNSFDBX 薄膜具有更长的激发态寿命. 根据文献报道 ${ }^{[19,20]}$ 可知, TCTA:CNSFDBX 和 TCTA:DCNSFDBX 的短寿命(衰 减)组分(分别为 $5.1 、 222.4 \mathrm{~ns}$ )来源于瞬时荧光，而长 寿命(衰减)组分(分别为 33.1、781.6 ns)则可能来源于 三重态激子的反系间䇀越一苂光发射.
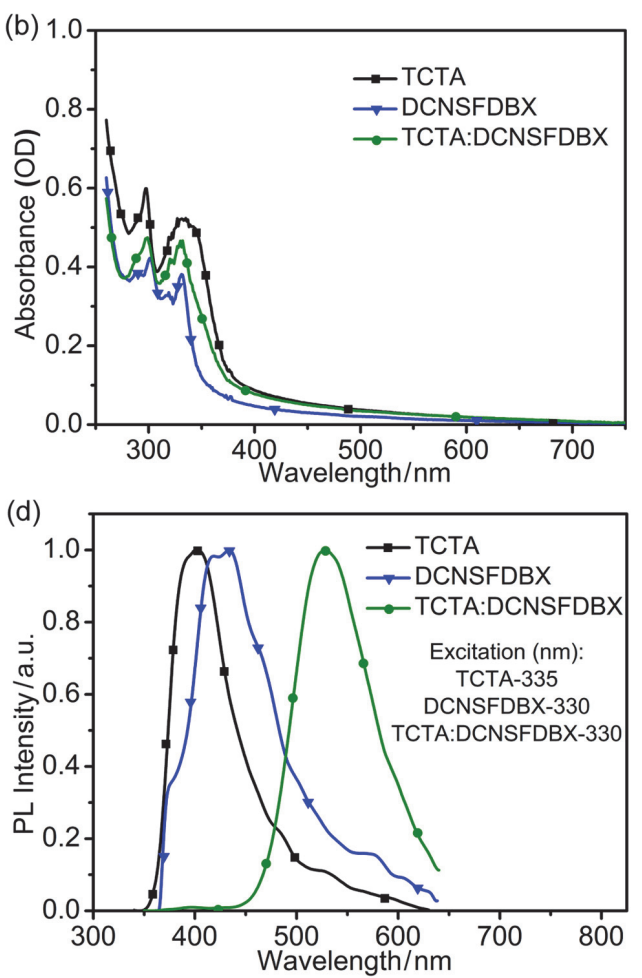

图 1 (a) TCTA, CNSFDBX, TCTA:CNSFDBX 的吸收光谱; (b) TCTA, DCNSFDBX, TCTA:DCNSFDBX 的吸收光谱; (c) TCTA, CNSFDBX, TCTA:CNSFDBX 的发射光谱; (d) TCTA, DCNSFDBX, TCTA:DCNSFDBX 的发射光谱(对应激发波长分别为: TCTA-335 nm, CNSFDBX-320 nm, DCNSFDBX-330 nm, TCTA:CNSFDBX-320 nm, TCTA:DCNSFDBX-330 nm).

Figure 1 UV-vis absorption spectra in the film-state: (a) TCTA, CNSFDBX and TCTA:CNSFDBX; (b) TCTA, DCNSFDBX and TCTA:DCNSFDBX. PL spectra in the film-state: (c) TCTA (excitation: $335 \mathrm{~nm}$ ), CNSFDBX (excitation: $320 \mathrm{~nm}$ ) and TCTA:CNSFDBX (excitation: $320 \mathrm{~nm}$ ); (d) TCTA (excitation: $335 \mathrm{~nm}$ ), DCNSFDBX (excitation: $330 \mathrm{~nm}$ ) and TCTA:DCNSFDBX (excitation: $330 \mathrm{~nm}$ ). 

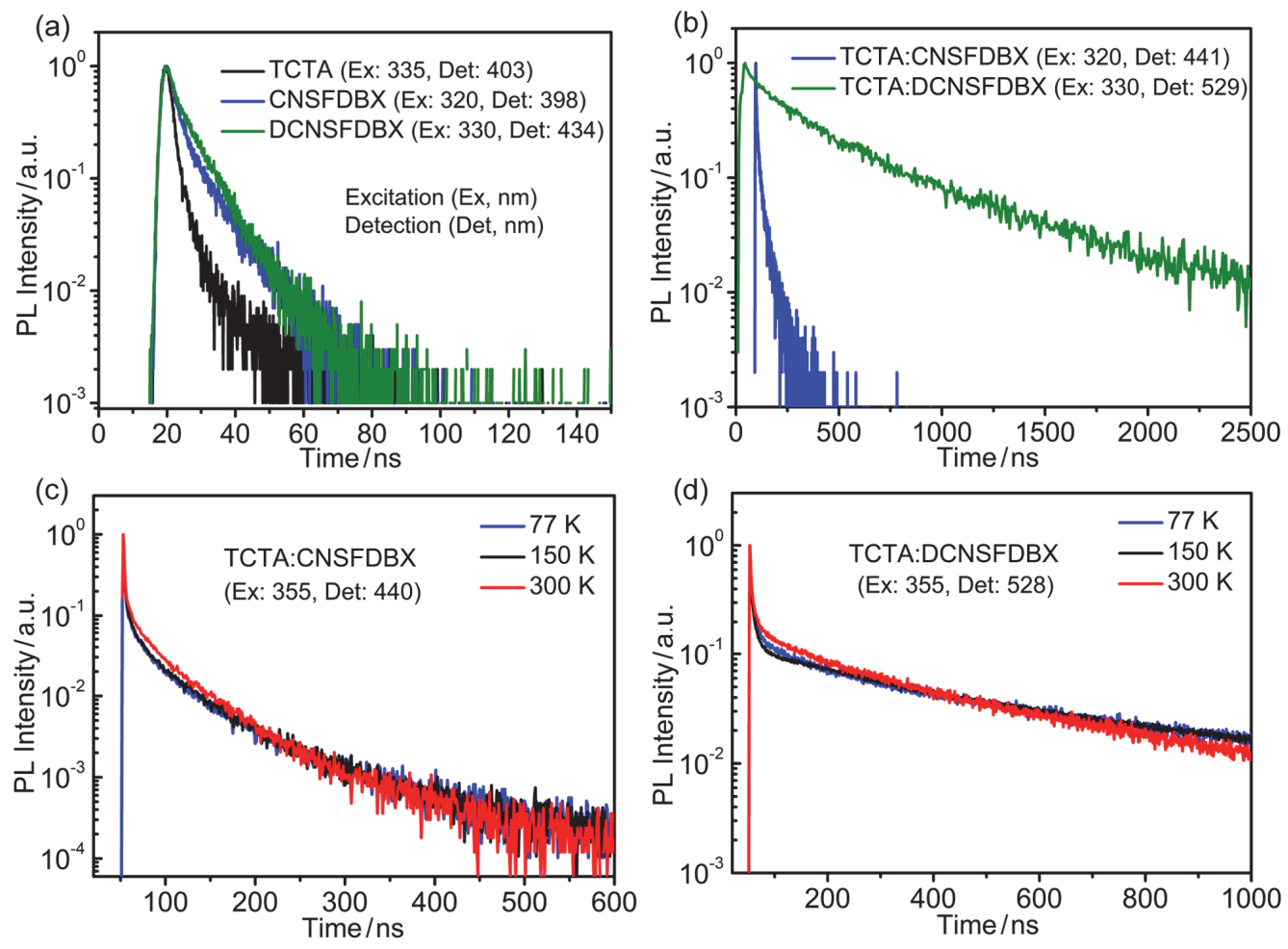

图 2 (a) TCTA, CNSFDBX 和 DCNSFDBX 薄膜的室温荧光衰减曲线(激发波长分别为 335、320、330 nm, 检测波长分别为 403、398、434 nm); (b) TCTA:CNSFDBX 和 TCTA:DCNSFDBX 薄膜的室温荧光衰减曲线(激发波长分别为 $320 、 330 \mathrm{~nm}$, 检测波长分别为 $441 、 529 \mathrm{~nm}$ ); (c) TCTA:CNSFDBX 和(d) TCTA:DCNSFDBX 薄膜的变温苂光衰减曲线(激发波长均为 $355 \mathrm{~nm}$, 检测波长分别为 $440 、 528 \mathrm{~nm}$ )

Figure 2 Fluorescence decays in the film-state at $300 \mathrm{~K}$ : (a) TCTA (excitation: $335 \mathrm{~nm}$, detection: $403 \mathrm{~nm}$ ), CNSFDBX (excitation: $320 \mathrm{~nm}$, detection: $398 \mathrm{~nm}$ ) and DCNSFDBX (excitation: $330 \mathrm{~nm}$, detection: $434 \mathrm{~nm}$ ); (b) TCTA:CNSFDBX (excitation: $320 \mathrm{~nm}$, detection: $441 \mathrm{~nm}$ ) and TCTA:DCNSFDBX (excitation: $330 \mathrm{~nm}$, detection: $529 \mathrm{~nm}$ ). Temperature-dependent transient fluorescence decays in the film-state: (c) TCTA:CNSFDBX (excitation: $355 \mathrm{~nm}$, detection: $440 \mathrm{~nm}$ ) and (d) TCTA:DCNSFDBX (excitation: $355 \mathrm{~nm}$, detection: $528 \mathrm{~nm}$ )

为了进一步判断掺杂体系的荧光发射是否具有延 迟苂光的性质, 我们对其进行了变温荧光衰减曲线测 试. 图 $2 \mathrm{c}, 2 \mathrm{~d}$ 和表格 $\mathrm{S} 2$ 显示, TCTA:CNSFDBX 和 TCTA:DCNSFDBX 的延迟衰减组分的荧光强度随温 度升高具有明显增加的特征. 根据文献报道 ${ }^{[37]}$, 这种 结果表明 TCTA:CNSFDBX 和 TCTA:DCNSFDBX 体系 具有明显的热活化延迟苂光发射的特征.

\section{4 低温荧光与低温磷光光谱表征}

为了更好地理解掺杂体系 TCTA:CNSFDBX 与 TCTA:DCNSFDBX 薄膜的激基复合物发光性质, 我们 对单体(TCTA, CNSFDBX, DCNSFDBX)和掺杂体系 (TCTA:CNSFDBX, TCTA:DCNSFDBX)的低温荧光光 谱和低温磷光光谱进行了测试. 其中, 对于化合物 DCNSFDBX 来讲, 我们只检测到了其低温荧光光谱. 相关结果已列于表格 S1、图 3 和图 S5 中.

根据图 S1, 3a 和 $3 \mathrm{~b}$ 结果计算得出, 单体 TCTA 的 $\mathrm{S}_{1}$ 态能级为 $3.20 \mathrm{eV}, \mathrm{T}_{1}$ 态能级为 $2.66 \mathrm{eV}$; CNSFDBX 的 $\mathrm{S}_{1}$ 态能级为 $3.19 \mathrm{eV}, \mathrm{T}_{1}$ 态能级为 $2.57 \mathrm{eV}$; DCNSFDBX 的 $\mathrm{S}_{1}$ 态能级为 $2.97 \mathrm{eV}$ (其低温磷光光谱未测得). 根据 图 3c 和 3d 结果计算得出, 掺杂体系 TCTA:CNSFDBX 的 $\mathrm{S}_{1}$ 态能级为 $2.82 \mathrm{eV}, \mathrm{T}_{1}$ 态能级为 $2.36 \mathrm{eV}$, 单线态 线态能隙差 $\Delta E_{\mathrm{ST}}$ 为 $0.46 \mathrm{eV}$; 而 TCTA:DCNSFDBX 的
$\mathrm{S}_{1}$ 态能级则为 $2.44 \mathrm{eV}, \mathrm{T}_{1}$ 态能级为 $2.32 \mathrm{eV}$, 单线态-三 线态能隙差 $\Delta E_{\mathrm{ST}}$ 为 $0.12 \mathrm{eV}$. 这种结果表明, 掺杂体系 TCTA:DCNSFDBX 的 $\mathrm{S}_{1}$ 与 $\mathrm{T}_{1}$ 态能级更接近, 更容易引 起反系间窝越过程 $\left(\mathrm{T}_{1} \rightarrow \mathrm{S}_{1}\right)$ 和延迟苂光的发生. 另外, 测试结果(表格 S1)显示, TCTA:DCNSFDBX 薄膜的光致 发光效率为 $29 \%$, 而 TCTA:CNSFDBX 薄膜的发光效率 为 $20 \%$. 显然, 这种较高的发光效率得益于 TCTA:DCNSFDBX 体系具有较小的 $\Delta E_{\mathrm{ST}}$ 值和 exciplex-TADF 特征. 当然, 这两种不同的 $\Delta E_{\mathrm{ST}}$ 值也造 成掺杂体系 TCTA:CNSFDBX 和 TCTA:DCNSFDBX 薄 膜具有不同的激发态寿命特征. 我们得到的苂光衰减测 试结果已经证明了这一点.

\section{5 电化学性质表征与量子化学计算}

为了深入认识掺杂体系 TCTA:CNSFDBX 和 TCTA:DCNSFDBX 薄膜激基复合物发光的形成过程, 我们进行了电化学性质测试和量子化学计算, 相关结果 已列于表格 S3 中. 根据早期文献 ${ }^{[24,26]}$ 报道, 激基复合物 发光的形成过程可与驱动力有关; 而驱动力 $\left(-\Delta G_{\mathrm{cs}}\right)$ 的 大小可由 Rehm-Weller 方程计算得出, 即 $-\Delta G_{\mathrm{cs}}$ 近似等 于单体分子的激发能 $\left(E_{\mathrm{A}^{*}}\right.$ 或 $E_{\mathrm{D}^{*}}$, 取较小的一个值使用) 与激基复合物的光学能带 $\left(E_{\text {exciplex }}\right)$ 的差值. 其中, $E_{\text {exciplex }}$ 的大小可由给体材料 TCTA 的氧化峰值减去受体材料 
(CNSFDBX 或 DCNSFDBX)的还原峰值得到. 根据表格 $\mathrm{S} 1$ 和 S3 可知, 掺杂体系 TCTA:CNSFDBX 的单体激发 能可由 CNSFDBX 的 $\mathrm{S}_{1}$ 态能级(3.19 eV)来表示, 其光学 能带 $E_{\text {exciplex }}$ (TCTA:CNSFDBX) 为 $2.97 \mathrm{eV}$, 因此 TCTA:CNSFDBX 形成过程的 $-\Delta G_{\mathrm{cs}}$ 值为 $0.22 \mathrm{eV}$. 对于 掺杂体系 TCTA:DCNSFDBX 来讲, 其单体激发能可由 DCNSFDBX 的 $\mathrm{S}_{1}$ 态能级 $(2.97 \mathrm{eV})$ 来表示, 其光学能带 $E_{\text {exciplex }}$ (TCTA:DCNSFDBX) 为 $2.58 \mathrm{eV}$, 因 此 TCTA:DCNSFDBX 形成过程的 $-\Delta G_{\mathrm{cs}}$ 值为 $0.39 \mathrm{eV}$. 由 此可见, TCTA:DCNSFDBX 形成过程的驱动力要大于 TCTA:CNSFDBX 形成过程的驱动力, 即更容易形成激 基复合物发光.

根据循环伏安测试结果(表 S3)计算得出, 单体分子 CNSFDBX 的最低分子空轨道(lowest unoccupied molecular orbital, LUMO)能级为 $-2.47 \mathrm{eV}$, 而 DCNSFDBX 的 LUMO 能级为 $-2.86 \mathrm{eV}$; 量子化学计算结果(表 $\mathrm{S} 3$ 和 S4)显示, CNSFDBX 和 DCNSFDBX 的 LUMO 能级分 别为 $-1.73 \mathrm{eV}$ 和 $-2.33 \mathrm{eV}$. 因此, 实验测试和理论计 算结果均表明, DCNSFDBX 具有比 CNSFDBX 更低的 LUMO 能级; 进一步说, SFX 骨架引入双㲵基取代使得 DCNSFDBX 比 CNSFDBX 具有更强的电子接受能力. 根据前期文献 ${ }^{[24,38]}$ 报道, 激基复合物形成的过程与给体 的给电子能力和受体的接受电子能力有关. 而且增加给
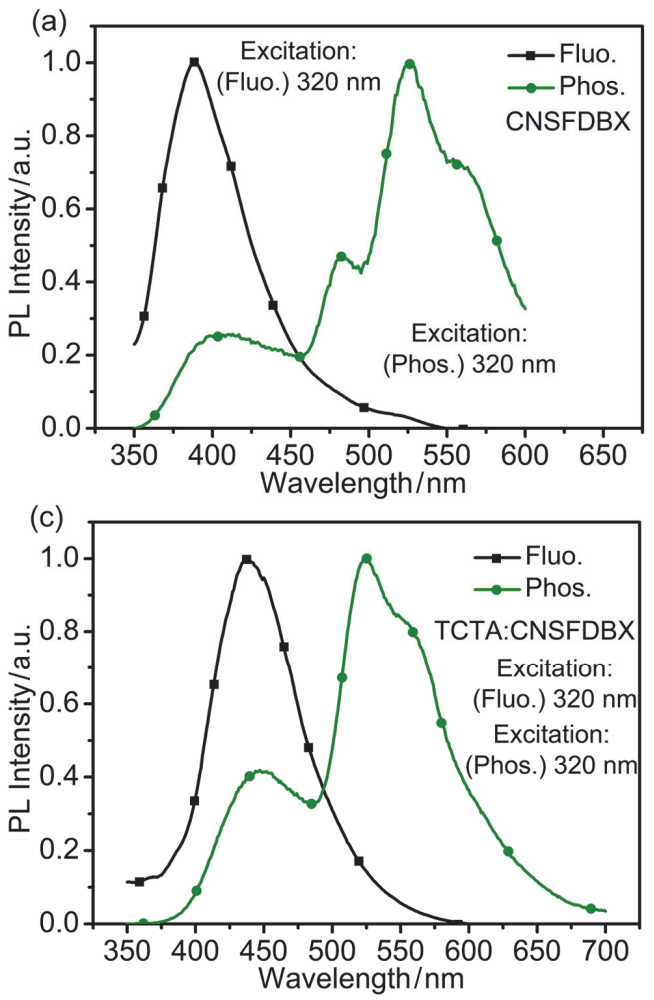

体的给电子能力或增加受体的接受电子能力将有助于 激基复合物的形成. 因此，相对于 CNSFDBX 分子结构 中的单氰基取代, DCNSFDBX 分子结构中的双氰基取 代使得该分子具有较强的电子接受能力，从而促使激基 复合物 TCTA:DCNSFDBX 更容易形成, 进而引起掺杂 体系 TCTA:DCNSFDBX 显示 exciplex-TADF 发射和较 高的光致发光效率.

\section{6 电致发光性质表征}

为了进一步研究掺杂体系 TCTA:CNSFDBX 和 TCTA:DCNSFDBX 的发光性质, 我们进行了发光器件 的制备与性能测试. 我们采用真空蒸镀的方法将 TCTA:CNSFDBX 和 TCTA:DCNSFDBX 直接作为发光 层制备出两个器件, 分别命名为 $\mathbf{A}$ 和 $\mathbf{B}$. 图 $4 \mathrm{a}$ 是这两个 器件的一般组成结构: (ITO) $/ \mathrm{MoO}_{3}(5 \mathrm{~nm}) / N, N^{\prime}-$ diphenyl- $N, N^{\prime}$-bis(1-naphthyl)(1,1'-biphenyl)-4,4'diamine (NPB, $35 \mathrm{~nm}) /$ TCTA $(5 \mathrm{~nm}) / \mathrm{TCTA}$ :CNSFX or DCNSFX (50 wt\%, $20 \mathrm{~nm}) / 1,3,5$-tri[(3-pyridyl)-phen-3-yl]benzene (TmPyPB, $40 \mathrm{~nm}) / \mathrm{LiF}(1 \mathrm{~nm}) / \mathrm{Al}(120 \mathrm{~nm})$. 制备器件 $\mathbf{A}$ 和 B 所需部分材料的化学结构已列于图 S6 中. 其中 $\mathrm{MoO}_{3}, \mathrm{NPB}, \mathrm{TCTA}, \mathrm{TmPyPB}$ 和 $\mathrm{LiF}$ 分别充当空穴注入 层、空穴传输层、电子/激子阻挡层、电子传输层和电子 注入层. 相关的测试数据已列于表格 S5 中.
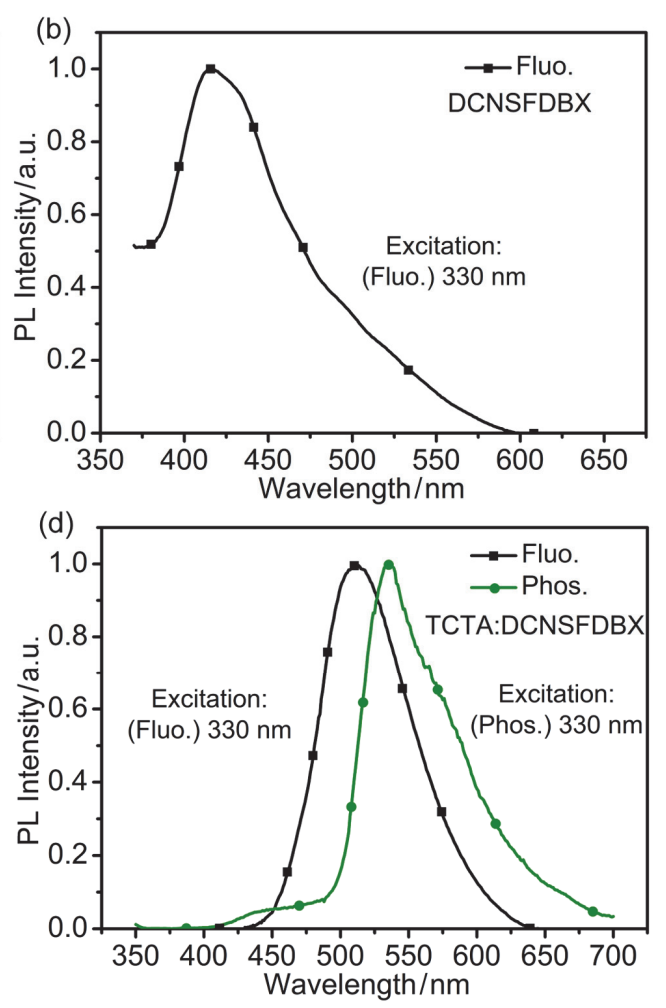

图 3 (a) CNSFDBX, (b) DCNSFDBX, (c) TCTA:CNSFDBX, (d) TCTA:DCNSFDBX 的低温苂光和低温磷光光谱(低温苂光和低温磷光使用相同的 激发波长, 分别为: CNSFDBX-320 nm, DCNSFDBX-330 nm, TCTA:CNSFDBX-320 nm, TCTA:DCNSFDBX-330 nm)

Figure 3 Film-state fluorescence and phosphorescence spectra of (a) CNSFDBX (excitation: $320 \mathrm{~nm}$ ), (b) DCNSFDBX (excitation: $330 \mathrm{~nm}$ ), (c) TCTA:CNSFDBX (excitation: $320 \mathrm{~nm}$ ) and (d) TCTA:DCNSFDBX (excitation: $330 \mathrm{~nm}$ ) at $77 \mathrm{~K}$ 




(c)







图 4 (a) 器件 $\mathbf{A}$ 和 $\mathbf{B}$ 的一般结构组成示意图; (b) 器件 $\mathbf{A}$ 和 $\mathbf{B}$ 在 $10 \mathrm{~V}$ 电压下的发光光谱; (c) 器件 $\mathbf{A}$ 和 $\mathbf{B}$ 的电流密度-电压-亮度曲线; (d) 器件 $\mathbf{A}$ 和 $\mathbf{B}$ 的电流效率-亮度-功率效率曲线

Figure 4 (a) The general structure, (b) EL spectra at $10 \mathrm{~V}$, (c) current density-voltage-luminance and (d) current efficiency-luminance-power efficiency characteristics for devices $\mathbf{A}$ and $\mathbf{B}$

图 $4 \mathrm{~b}$ 显示, 器件 $\mathbf{A}$ 和 $\mathbf{B}$ 在 $10 \mathrm{~V}$ 的电压驱动作用下 的电致发光光谱峰位分别位于 $452 \mathrm{~nm}$ 和 $504 \mathrm{~nm}$, 对应 的 CIE (Commission International de l'Eclairage)色坐标分 别为 $(0.17,0.18)$ 和 $(0.33,0.52)$. 另外, 器件 $\mathbf{A}$ 和 $\mathbf{B}$ 的电致 发光光谱形状与掺杂薄膜的光致发光谱的类似, 由此可 见器件 $\mathrm{A}$ 和 $\mathrm{B}$ 的发光分别来自掺杂体系 TCTA:CNSFDBX 和 TCTA:DCNSFDBX 的 exciplex 发 射. 图 $4 \mathrm{c}$ 显示, 器件 $\mathbf{A}$ 和 $\mathbf{B}$ 的启动电压均为 $4.8 \mathrm{~V}$, 最 大发光亮度分别为 $4956 \mathrm{~cd} \cdot \mathrm{m}^{-2}$ 和 $6460 \mathrm{~cd} \cdot \mathrm{m}^{-2}$. 显然, 器件 B 所表现的较高亮度得益于 TCTA:DCNSFDBX 薄 膜较好的光致发光性能.

图 4d 显示, 器件 $\mathbf{A}$ 和 $\mathbf{B}$ 具有不同的电致发光效率, 其中 $\mathbf{A}$ 的最大电流效率 $\eta_{\mathrm{c}}$ 和功率效率 $\eta_{\mathrm{p}}$ 分别为 $4.0 \mathrm{~cd}$ $\mathrm{A}^{-1}$ 和 $2.0 \mathrm{~cd} \cdot \mathrm{A}^{-1}, \mathbf{B}$ 的最大电流效率 $\eta_{\mathrm{c}}$ 和功率效率 $\eta_{\mathrm{p}}$ 分别为 $5.7 \mathrm{~cd} \cdot \mathrm{A}^{-1}$ 和 $3.1 \mathrm{~cd} \cdot \mathrm{A}^{-1}$. 另外, 表格 S5 显示器 件 $\mathbf{B}$ 仍表现出高于器件 $\mathbf{A}$ 的外量子效率, 其数值分别为 $1.6 \%$ 和 $1.0 \%$. 显然, 器件 B 表现出的相对高的发光效率 可归因于 TCTA:DCNSFDBX 薄膜更容易实现 exciplex-TADF 特征发射, 尽管其外量子效率低于 5\%. 值得一提的是, 表格 $\mathrm{S} 1$ 和 $\mathrm{S} 3$ 显示单体 TCTA 的 $\mathrm{T}_{1}$ 态能 级为 $2.66 \mathrm{eV}, \mathrm{CNSFDBX}$ 的 $\mathrm{T}_{1}$ 态能级为 $2.57 \mathrm{eV}$, 均低于 掺杂体系 TCTA:CNSFDBX 的光学能带 $E_{\text {exciplex }}$ (2.97 $\mathrm{eV}$ ). 因此, TCTA:CNSFDBX 受到外界能量激发后(如电 场作用、光激发等)向单体分子 TCTA 或 CNSFDBX 发 生能量传递(流失)存在较大的可能性, 并进一步导致
TCTA:CNSFDBX 体系显示较低的光致发光效率和电致 发光效率. 另外, 这种潜在的能量流失则可能会影响器 件效率的稳定性, 即效率曲线出现波动. 因此, 图 $4 \mathrm{~d}$ 中 器件 $\mathbf{A}$ 的效率曲线出现的波动则可能是由 TCTA:CNSFDBX 向 TCTA 或 CNSFDBX 发生潜在的能 量传递(流失)造成的. 相反, DCNSFDBX 的 $\mathrm{S}_{1}$ 态能级为 $2.97 \mathrm{eV}$ ( $\mathrm{T}_{1}$ 态能级未测得), 高于掺杂体系 TCTA:DCNSFDBX 的光学能带 $E_{\text {exciplex }}(2.58 \mathrm{eV})$. 因此, TCTA:DCNSFDBX 向单体分子 TCTA 或 DCNSFDBX 发 生能量传递(流失)的可能性很低, 因而能够较好地保证 激子进行 exciplex (TCTA:DCNSFDBX)发射, 进而显示 较高的发光效率.

\section{3 结论}

本工作设计并合成了两个基于 SFX 的新型电子受 体材料 CNSFDBX 和 DCNSFDBX, 经过与主体材料 TCTA 的掺杂, 实现了激基复合物发光. 其中, 掺杂体 系 TCTA:DCNSFDBX 表现出优于 TCTA:CNSFDBX 的 发光性能, 对应的光致发光效率分别为 $29 \%$ 和 $20 \%$, 电 流效率分别为 5.7 和 $4.0 \mathrm{~cd} \cdot \mathrm{A}^{-1}$, 功率效率分别为 3.1 和 $2.0 \mathrm{~cd} \cdot \mathrm{A}^{-1}$, 外量子效率分别为 $1.6 \%$ 和 $1.0 \%$. 这种 较高的发光性能归因于双氧基取代使得 DCNSFDBX 具 有更强的接受电子的能力, 引起 TCTA 与 DCNSFDBX 更容易形成激基复合物并显示更小的单线态-三线态能 
隙差，进而促进 TCTA:DCNSFDBX 体系更容易发生反 系间窝越过程 $\left(\mathrm{T}_{1} \rightarrow \mathrm{S}_{1}\right)$ 和 TADF 发射. 本工作为今后开 发面向 exciplex-TADF 应用的新型电子受体材料提供了 思路, 相关的研究已在我们课题组进行了展开.

\section{4 实验部分}

\section{1 试剂与测试仪器}

本工作所使用的试剂均为分析纯, 部分试剂在使用 前通过蒸馏得到了进一步纯化. 采用 Bruker Avance II DMX 400 型核磁共振仪进行 ${ }^{1} \mathrm{H}$ NMR 和 ${ }^{13} \mathrm{C}$ NMR 谱图 的测试( $\mathrm{CDCl}_{3}$ 作溶剂), 借助 Shimadzu GCMS 2010 PLUS 型质谱仪进行化合物分子量的测定, 利用 Elementa Analysensysteme GmbH Vario EL III 型元素分 析仪对化合物进行元素分析测定. 实验中采用 Shimadzu UV-3150 型 UV-Vis-NIR 光谱仪进行吸收光谱 的测试, 采用 Shimadzu RF-530XPC 型苂光光谱仪进行 室温发射光谱测试, 采用 Hitachi F-4600 型苂光光谱仪 进行低温苂光和低温磷光光谱的测试, 采用 Edinburgh Instruments FLS920 型多功能荧光光谱仪进行室温苂光 衰减曲线和发光量子产率的测试, 采用 TimeHarp 260 型时间相关单光子计数仪进行变温荧光衰减曲线的测 试.

\section{2 电化学性质测试}

本工作利用 CHI660E 电化学工作站于室温下进行 循环伏安曲线的测试, 并采用三电极工作体系(玻碳电 极为工作电极, 铂丝电极为对电极, $\mathrm{Ag} / \mathrm{AgNO}_{3}$ 电极为参 比电极). 循环伏安测试采用四丁基六氟磷酸铵溶液(溶 剂为 $\mathrm{CH}_{2} \mathrm{Cl}_{2}$, 溶液浓度为 $0.1 \mathrm{~mol} \cdot \mathrm{L}^{-1}$ ) 为电解质(氮气氛 围), 测试扫描(电压)频率为 $0.1 \mathrm{~V} \cdot \mathrm{s}^{-1}$.

\section{3 样品薄膜与器件的制备}

采用真空蒸镀方式制备样品薄膜用于光致发光性 质测试, 其中掺杂薄膜中给、受体材料的质量比为 $1: 1$. 薄膜制备的具体过程为: 分别取 TCTA、CNSFDBX、 DCNSFDBX 各 $20 \mathrm{mg}$ 置于蒸镀室内, 用电流大小控制 蒸发速率. 对于每一种单体薄膜(TCTA、CNSFDBX、 DCNSFDBX)来讲，控制蒸镀速率在 $0.1 \sim 1.5 \AA \cdot \mathrm{s}^{-1}$ 范围 内, 稳定在 $1.3 \AA \cdot \mathrm{s}^{-1}$, 将每个单体在石英片上分别蒸镀 厚度为 $30 \mathrm{~nm}$, 分别得到 3 个石英片薄膜(TCTA、 CNSFDBX 、 DCNSFDBX). 对于掺杂薄 膜 (TCTA:CNSFDBX、TCTA:DCNSFDBX)体系来讲，控制 TCTA 和 CNSFDBX(DCNSFDBX)的蒸镀速率相同(蒸镀 速率控制在 $0.1 \sim 1.5 \AA \cdot \mathrm{s}^{-1}$ 范围内, 稳定在 $\left.1.3 \AA \cdot \mathrm{s}^{-1}\right)$, 进行 1:1 混合蒸镀, 将 TCTA 和 CNSFDBX(DCNSFDBX) 在同一石英片上同时各蒸镀 $15 \mathrm{~nm}$, 厚度总为 $30 \mathrm{~nm}$, 分 别得到 2 个石英片薄膜 (TCTA:CNSFDBX 、 TCTA:DCNSFDBX).

本工作选用 ITO 玻璃祄底(方块电阻为 $10 \Omega \cdot \mathrm{sq}^{-1}$
欧姆/平方)经清洗液清洗 $10 \mathrm{~min}$, 然后用去离子水清洗 祄底上的洗涤剂. 紧接着, 使用丙酮和异丙醇对玻璃衬 底进行进一步清洗, 然后使用超声以除去其上面的杂 质. 随后，用惰性气体吹干玻璃衬底并放入干燥箱进行 干燥. 最后, 我们把玻璃祄底放入紫外腔中进行紫外臭 氧处理 $5 \mathrm{~min}$. 前期准备工作结束之后, 我们把 ITO 玻 璃祄底放进真空腔内, 采用有机气相沉积系统进行材料 的蒸镀, 腔内气压设置为 $5 \times 10^{-4} \mathrm{~Pa}$, 发光器件的面积 约为 $4 \mathrm{~mm} \times 4 \mathrm{~mm}$. 我们采用 Keithley 2400 电流源测试 器件的电学特性, 使用 PR655 光谱仪测试器件的光谱. 器件制备的具体过程为: 分别取 TmPyPB、NPB、TCTA、 CNSFDBX、DCNSFDBX、 LiF(各 $20 \mathrm{mg}$ )及铝条放置于 蒸镀室内, 用电流大小控制蒸发速率. 按照设计好的器 件结构进行蒸镀, 其中有机物的蒸镀速率控制在 $0.1 \sim$ $1.5 \AA \cdot \mathrm{s}^{-1}$ 范围, 稳定在 $1.3 \AA \cdot \mathrm{s}^{-1}$; 无机物的蒸镀速率控 制在 $0.05 \sim 0.3 \AA \cdot \mathrm{s}^{-1}$ 范围; 铝的蒸镀速率大于 $3 \AA \cdot \mathrm{s}^{-1}$, 稳定在 $8 \sim 10 \AA \cdot \mathrm{s}^{-1}$. 对于 TCTA:CNSFDBX 和 TCTA:DCNSFDBX 来讲, 控制 TCTA 和 CNSFDBX (DCNSFDBX) 的蒸镀速率相同, 进行 1:1 混合蒸镀, 将 TCTA 和 CNSFDBX(DCNSFDBX)在同一石英片上同时 各蒸镀 $10 \mathrm{~nm}$, 厚度总为 $20 \mathrm{~nm}$, 最后分别得到器件 $\mathbf{A}$ 和 B.

\section{4 化合物的合成}

\subsubsection{2-氰基螺芴苯并氧杂葱(CNSFDBX)的合成}

取一个干燥的 $150 \mathrm{~mL}$ 的两口烧瓶, 依次加入 2-溴 螺芴苯并氧杂蒽 $(0.50 \mathrm{~g}, 0.98 \mathrm{mmol})$, 亚铁氰化钾 $(0.41$ $\mathrm{g}, 0.98 \mathrm{mmol})$, 醋酸钯 $(0.1 \sim 0.5 \mathrm{~mol} \%)$, 碳酸钠 $(0.10 \mathrm{~g}$, $0.98 \mathrm{mmol})$. 利用锡纸避光, 对混合体系抽真空充氮气 三次, 然后用注射器向反应体系加入 $N, N$-二甲基甲酰胺 溶剂(DMF, $10 \mathrm{~mL})$. 加热至 $160{ }^{\circ} \mathrm{C}$, 回流摚拌反应 $24 \mathrm{~h}$. 反应结束后，将混合体系冷却至室温，向反应瓶中加入 二氯甲烷 $(40 \mathrm{~mL})$, 随后将混合液倒入 $500 \mathrm{~mL}$ 的分液漏 斗中, 加入水进行萃取三次. 有机相用无水硫酸美干燥, 然后对有机相进行硅胶柱色谱分离, 得到浅黄色固体 CNSFDBX $(0.38 \mathrm{~g})$, 产率约为 $85 \%$. GC-MS (EI- $m / z)$ : 理 论值: 357.12, 实测值: 357. 元素分析: 理论值为: C, 89.26; H, 4.19; N, 3.06; O, 3.50. 实测值: C, 89.29; H, 4.06; N, 3.09; O, 3.56. ${ }^{1} \mathrm{H}$ NMR (400 MHz, $\left.\mathrm{CDCl}_{3}\right) \delta: 8.83$ $(\mathrm{d}, J=8.0 \mathrm{~Hz}, 2 \mathrm{H}), 7.96(\mathrm{t}, J=8.0 \mathrm{~Hz}, 2 \mathrm{H}), 7.70 \sim 7.81$ $(\mathrm{m}, 5 \mathrm{H}), 7.61(\mathrm{t}, J=8.0 \mathrm{~Hz}, 2 \mathrm{H}), 7.49(\mathrm{t}, J=8.0 \mathrm{~Hz}, 2 \mathrm{H})$, $7.30 \sim 7.34(\mathrm{~m}, 3 \mathrm{H}), 7.24(\mathrm{~d}, J=8.0 \mathrm{~Hz}, 1 \mathrm{H}), 6.37(\mathrm{~d}, J=$ $8.0 \mathrm{~Hz}, 2 \mathrm{H}) .{ }^{13} \mathrm{C} \mathrm{NMR}\left(100 \mathrm{MHz}, \mathrm{CDCl}_{3}\right) \delta: 156.39$, $146.00,144.22,138.03,133.72,132.41,130.42,130.15$, $128.60,127.77,127.06,126.62,124.85,124.65,123.52$, $121.80,121.07,120.73,118.96,116.46,111.47,54.51$.

4.4 2, 7 -二氧基螺芴苯并氧杂蒽(DCNSFDBX)的合成 取一个干燥的 $150 \mathrm{~mL}$ 的两口烧瓶, 依次加入 2,7- 
二溴螺芴苯并氧杂葱 $(0.50 \mathrm{~g}, 0.85 \mathrm{mmol})$, 亚铁氰化钾 $(0.36 \mathrm{~g}, 0.85 \mathrm{mmol})$, 醋酸钯 $(0.1 \sim 0.5 \mathrm{~mol} \%)$, 碳酸钠 (0.09 g, $0.85 \mathrm{mmol})$. 利用锡纸避光, 对混合体系抽真空 充氮气三次, 然后用注射器向反应体系加入 $N, N$-二甲基 甲酰胺溶剂(DMF, $10 \mathrm{~mL})$. 加热至 $160{ }^{\circ} \mathrm{C}$, 回流摚拌反 应 $24 \mathrm{~h}$. 反应结束后, 将混合体系冷却至室温, 向反应 瓶中加入二氯甲烷 $(40 \mathrm{~mL})$, 随后将混合液倒入 $500 \mathrm{~mL}$ 的分液漏斗中, 加入水进行萃取三次. 有机相用无水硫 酸镁干燥, 然后对有机相进行硅胶柱色谱分离, 得到浅 黄色固体 DCNSFDBX (0.32 g), 产率约为 78\%. GC-MS (EI- $m / z)$ : 理论值: 482.14, 实测值: 482. 元素分析: 理论 值为: $\mathrm{C}, 87.12 ; \mathrm{H}, 3.76 ; \mathrm{N}, 5.81 ; \mathrm{O}, 3.32$. 实测值: $\mathrm{C}$, 87.19; H, 3.73; N, 5.76; O, 3.32. ${ }^{1} \mathrm{H}$ NMR (400 MHz, $\left.\mathrm{CDCl}_{3}\right) \delta: 8.82(\mathrm{~d}, J=8.0 \mathrm{~Hz}, 2 \mathrm{H}), 8.03(\mathrm{~d}, J=8.0 \mathrm{~Hz}$, $2 \mathrm{H}), 7.76 \sim 7.82(\mathrm{~m}, 6 \mathrm{H}), 7.63(\mathrm{t}, J=4.0 \mathrm{~Hz}, 2 \mathrm{H}), 7.52(\mathrm{~s}$, $2 \mathrm{H}), 7.35(\mathrm{~d}, J=8.0 \mathrm{~Hz}, 2 \mathrm{H}), 6.29(\mathrm{~d}, J=8.0 \mathrm{~Hz}, 2 \mathrm{H}) .{ }^{13} \mathrm{C}$ NMR (100 MHz, $\left.\mathrm{CDCl}_{3}\right) \delta: 156.76,146.09,142.07$, $133.87,132.70,130.39,127.81,127.45,126.94,124.65$, $124.10,123.93,121.85,118.36,114.76,113.41,54.80$.

\section{References}

[1] Tang, C. W.; VanSlyke, S. A. Appl. Phys. Lett. 1987, 51, 913.

[2] Baldo, M. A.; O'Brien, D. F.; You, Y.; Shoustikov, A.; Sibley, S.; Thompson, M. E.; Forrest, S. R. Nature 1998, 395, 151.

[3] Kido, J.; Kimura, M.; Nagai, K. Science 1995, 267, 1332.

[4] Yu, J.; Xiao, Y.; Chen, J. Chin. J. Org. Chem. 2019, 39, 3460 (in Chinese). (俞佳, 肖雅方, 陈嘉雄, 有机化学, 2019, 39, 3460.)

[5] Zhang, T.; Qiu, Z.; Cheng, X.; Zhang, Y.; Wang, X. Chin. J. Org. Chem. 2019, 39, 2534 (in Chinese). (张婷, 邱子夜, 程肖杰, 张雨 露, 汪徐春, 有机化学, 2019, 39, 2534.)

[6] Wang, T.; Hua, X.; Yu, Y.; Yuan, Y.; Feng, M.; Jiang, Z. Chin. J. Org. Chem. 2019, 39, 1436 (in Chinese). (王形彫, 华晓晨, 郁友军, 袁 熠, 冯敏强, 蒋佐权, 有机化学, 2019, 39, 1436.)

[7] He, X.; Xiao, Y.; Yuan, X.; Ye, S.; Jiang, H. Chin. J. Org. Chem. 2019, 39, 761 (in Chinese). (何煦, 肖燏萍, 袁金否, 叶尚辉, 姜鸿 基, 有机化学, 2019, 39, 761.)

[8] Wang, F.; Cao, X.; Mei, L.; Zhang, X.; Hu, J.; Tao, Y. Chinese J. Chem. 2018, 36, 241.

[9] Lin, D.; Song, S.; Chen, Z.; Guo, P.; Chen, J.; Shi, H.; Mai, Y.; Song, H. Chin. J. Org. Chem. 2018, 38, 103 (in Chinese). (林丹燕, 宋森 川, 陈智勇, 郭鹏然, 陈江韩, 史华红, 麦裕良, 宋化灿, 有机化 学, 2018, 38, 103.)

[10] Li, X.; Zhang, J.; Zhao, Z.; Wang, L.; Yang, H.; Chang, Q.; Jiang, N.; Liu, Z.; Bian, Z.; Liu, W.; Lu, Z.; Huang, C. Adv. Mater. 2018, 30,1705005 .
[11] Klimes, K.; Zhu, Z.-Q.; Li, J. Adv. Funct. Mater. 2019, 29, 1903068.

[12] Kim, K.-H.; Kim, J.-J. Adv. Mater. 2018, 30, 1705600.

[13] You, Y.; Park, S. Y. Dalton Trans. 2009, 1267.

[14] Cheng, G.; Kwak, Y.; To, W.-P.; Lam, T.-L.; Tong, G. S. M.; Sit, M.-K.; Gong, S.; Choi, B.; Choi, W. i.; Yang, C.; Che, C.-M. ACS Appl. Mater. Interfaces 2019, 11, 45161.

[15] Cao, H.; Shan, G.; Wen, X.; Sun, H.; Su, Z.; Zhong, R.; Xie, W.; Li, P.; Zhu, D. J. Mater. Chem. C 2013, 1,7371 .

[16] Endo, A.; Ogasawara, M.; Takahashi, A.; Yokoyama, D.; Kato, Y.; Adachi, C. Adv. Mater. 2009, 21, 4802.

[17] Tao, Y.; Yuan, K.; Chen, T.; Xu, P.; Li, H.; Chen, R.; Zheng, C.; Zhang, L.; Huang, W. Adv. Mater. 2014, 26, 7931.

[18] Liu, Y.; Li, C.; Ren, Z.; Yan, S.; Bryce, M. R. Nat. Rev. Mater. 2018, 3,18020 .

[19] Nakagawa, T.; Ku, S.-Y.; Wong, K.-T.; Adachi, C. Chem. Commun. 2012, 48,9580 .

[20] Tanaka, H.; Shizu, K.; Miyazaki, H.; Adachi, C. Chem. Commun. 2012, 48, 11392.

[21] Wong, M. Y.; Zysman-Colman, E. Adv. Mater. 2017, 29, 1605444.

[22] Sarma, M.; Wong, K.-T. ACS Appl. Mater. Interfaces 2018, 10, 19279 .

[23] Ng, T.-W.; Lo, M.-F.; Fung, M.-K.; Zhang, W.-J.; Lee, C.-S. Adv. Mater. 2014, 26, 5569.

[24] Goushi, K.; Yoshida, K.; Sato, K.; Adachi, C. Nat. Photon. 2012, 6, 253.

[25] Li, J.; Nomura, H.; Miyazaki, H.; Adachi, C. Chem. Commun. 2014, $50,6174$.

[26] Liu, X.-K.; Chen, Z.; Zheng, C.-J.; Liu, C.-L.; Lee, C.-S.; Li, F.; Ou, X.-M.; Zhang, X.-H. Adv. Mater. 2015, 27, 2378.

[27] Zhang, M.; Liu, W.; Zheng, C.-J.; Wang, K.; Shi, Y.-Z.; Li, X.; Lin, H.; Tao, S.-L.; Zhang, X.-H. Adv. Sci. 2019, 6, 1801938.

[28] Chapran, M.; Pander, P.; Vasylieva, M.; Wiosna-Salyga, G.; Ulanski, J.; Dias, F. B.; Data, P. ACS Appl. Mater. Interfaces 2019, 11, 13460.

[29] Cao, H.-T.; Zhao, Y.; Sun, C.; Fang, D.; Xie, L.-H.; Yan, M.-N.; Wei, Y.; Zhang, H.-M.; Huang, W. Dyes Pigm. 2018, 149, 422.

[30] Cao, H.-T.; Hong, C.-S.; Ye, D.-Q.; Liu, L.-H.; Xie, L.-H.; Chen, S.-F.; Sun, C.; Wang, S.-S.; Zhang, H.-M.; Huang, W. J. Mol. Struct. 2019, 1196, 132.

[31] Xie, L.-H.; Liu, F.; Tang, C.; Hou, X.-Y.; Hua, Y.-R.; Fan, Q.-L.; Huang, W. Org. Lett. 2006, 8, 2787.

[32] Ou, C.-J.; Ren, B.-Y.; Li, J.-W.; Lin, D.-Q.; Zhong, C.; Xie, L.-H.; Zhao, J.-F.; Mi, B.-X.; Cao, H.-T.; Huang, W. Org. Electron. 2017, $43,87$.

[33] Ou, C.-J.; Zhu, C.; Ding, X.-H.; Yang, L.; Lin, J.-Y.; Xie, L.-H.; Qian, Y.; Xu, C.-X.; Zhao, J.-F.; Huang, W. J. Mater. Chem. C 2017, 5,5345 .

[34] Iwata, S.; Tanaka, J.; Nagakura, S. J. Chem. Phys. 1967, 47, 2203.

[35] Gould, I. R.; Young, R. H.; Mueller, L. J.; Farid, S. J. Am. Chem. Soc. 1994, 116, 8176 .

[36] Kalinowski, J.; Giro, G.; Cocchi, M.; Fattori, V.; Di Marco, P. Appl. Phys. Lett. 2000, 76, 2352.

[37] Uoyama, H.; Goushi, K.; Shizu, K.; Nomura, H.; Adachi, C. Nature 2012, 492, 234.

[38] Wu, T.-L.; Liao, S.-Y.; Huang, P.-Y.; Hong, Z.-S.; Huang, M.-P.; Lin, C.-C.; Cheng, M.-J.; Cheng, C.-H. ACS Appl. Mater. Interfaces 2019, 11, 19294. 